\title{
Key for Successful Home-Based Slow Oral Immunotherapy in Children with Egg Allergy: Age and Sensitivity
}

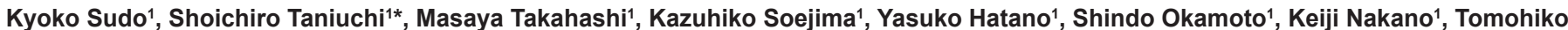
Shimo', Hayato Koshino² and Kazunari Kaneko ${ }^{1}$

${ }^{1}$ Department of Pediatrics, Kansai Medical University, Japan

${ }^{2}$ Brain Inc., Kyoto, Japan

\begin{abstract}
Background: Oral immunotherapy (OIT) has been recognized as a promising therapy for children with food allergy. However a part of applied patients was not able to increase doses of allergic food. Since a clear standard for the appropriate patient selection for OIT has not been established, we tried to determine the indication criteria for when and/or to whom OIT should be applied.

Method: We retrospectively reviewed the medical records of 82 children treated by home-based slow OIT which shows the administration of baked eggs orally every 2-3 days at home for two months as maintenance dose after open food challenge and statistically analyzed their clinical courses to identify the prognostic factors associated with a successful result. We applied the variables such as age, sex, symptoms during OIT, initial dose when starting OIT, specific IgE level and dislike of eggs, to a multivariate stepwise logistic regression analysis.

Results: A total of $40(56 \%)$ children reached remission in the OIT trial for a median of 213 days without severer allergic reactions, the remaining $31(44 \%)$ could not reach the remission stage. We found the clinical course of the patients to be significantly associated with age and the initial dose of OIT. The children under 4 years of age or who could ingest larger portions (more than $1 \mathrm{~g}$ if under 4 years, and $6 \mathrm{~g}$ if over 5 years) as an initial dose were found to be the best candidates for OIT.

Conclusions: This study revealed that home-based slow OIT caused almost successful results without severe allergic results in the children with egg allergy, and especially when starting before 5 years of age it is remarkable even if their initial doses were limited. This is the first report to contribute to the establishment of the indication criteria for home-based OIT.
\end{abstract}

Keywords: Egg allergy; Indication criteria; Prognostic factor; Oral immunotherapy

\section{Introduction}

Egg allergy is one of the most frequent food allergies in Japan affecting about 1 to $5 \%$ of young children [1]. Common symptoms of egg allergy involve the skin as well as the digestive tract, and in more severe cases, can also result in anaphylaxis. Although two- thirds or more of these children outgrow the allergy by around 6 years of age [1-3], one- third of the population of children continues to suffer from persistent allergic reactions. Oral immunotherapy (OIT) is a promising therapy for food allergies, especially for IgE-mediated cow's milk and hen's egg allergies, during childhood [1,4-12].

Most of OIT regimens include weekly dose increases in the hospital setting, with dose maintenance during the week, taking from 12 to 24 weeks (or even more) to reach the final dose. Recently, schedules with a duration of only a few days have been introduced but they require that the treated patient be admitted to the hospital or at least attend the hospital daily because of frequent severe adverse reactions.

We designed new protocol for more safety and avoiding inconvenience of both children and their parents in the children with egg allergy. The new protocol is more slowly increased dose than conventional OIT regimens and the children ingest only 3 days per week.

In the past two years, we have applied the new protocol of OIT (so called home-based slow OIT) to young children with egg allergy. In our experiences, a certain population of children still showed a persistence of their egg allergy, although the majority of children obtained favorable results (unpublished data). As Kurihara has reviewed, it might be important to clarify the indication criteria with regard to which type of patients each therapy should be applied [1]. In the present study, we have statistically analyzed the outcomes of home-based OIT for children with egg allergy to elucidate the prognostic factors for this therapy.

\section{Patients and Methods}

Home-based slow OIT was conducted on 82 children (53 males and 29 females) at a median age of 5 years (interquartile range (IQR), 3 years) who had been diagnosed with egg allergy by a food challenge test at Kansai Medical University, Osaka, Japan. The survey was retrospectively carried out based on the medical records of these children. We statistically analyzed the clinical course of the therapy to elucidate the prognostic factors for a successful result.

\section{Oral food challenge test}

All egg-food challenges were open challenges, performed in

*Corresponding author: Shoichiro Taniuchi, Department of Pediatrics, Kansai Medical University, 10-15 Fumizono-cho, Moriguchi-shi, Osaka 570-8506, Japan Tel: (+81)-6-6992-1001; Fax: (+81)-6-6992-9355; E-mail: taniuchi@takii.kmu.ac.jp

Received April 17, 2013; Accepted May 17, 2013; Published May 23, 2013

Citation: Sudo K, Taniuchi S, Takahashi M, Soejima K, Hatano Y, et al. (2013) Key for Successful Home-Based Slow Oral Immunotherapy in Children with Egg Allergy: Age and Sensitivity. J Allergy Ther S3: 009. doi:10.4172/2155-6121.S3-009

Copyright: @ 2013 Sudo K, et al. This is an open-access article distributed under the terms of the Creative Commons Attribution License, which permits unrestricted use, distribution, and reproduction in any medium, provided the original author and source are credited. 
hospital settings and supervised by physicians in accordance with the guidelines for diagnosis and management of pediatric food allergy in Japan [13]. During the challenge, full emergency equipment was at hand. The children's parents prior to enrolment in the study gave informed consent. Patients taking anti-histamines were asked to avoid them at least $48 \mathrm{~h}$ before provocation but topical steroids were authorized, and were admitted to our day-clinic in the morning in a fasting state. Challenge material was cooked egg baked for $3 \mathrm{~min}$ and then steamed for $10 \mathrm{~min}$ for open challenges. The initial challenge dose and the following doses were set according to the history of the last reaction, but were similar in most patients. If the patient tolerated the first dose, subsequent doses were given every $30 \mathrm{~min}$. Doses and time intervals between them were adjusted in accordance with symptoms observed during the challenges. The challenge was interrupted if children demonstrated unambiguous clinical reactivity or after the administration of $45 \mathrm{~g}$ of egg. All children were then observed for at least three more hours after the end of the feeding.

\section{Home-based slow OIT}

All the subjects were outpatients. We used baked egg as previously described. The initial dose of home based slow OIT was set to be a sub threshold dose, usually one fourth or one eighth of the threshold dose determined during a preliminary open challenge test. The initial dose, if no adverse reactions existed, was administered orally every 2-3 days at home for two months as a maintenance dose. The children were challenged to ingest a double dose under medical supervision at every two-month follow-up visit. They repeated the escalation phase in the same manner until being able to ingest a half of an egg, which was defined as a remission dose. They were encouraged to continue ingesting a further increased dose in a less cooked condition even after reaching the remission dose.

\section{Laboratory test}

Blood samples were collected before starting OIT, during the remission stage or after 6 months if remission was not reached, and 12 months after starting OIT. They were analyzed for egg white-specific serum IgE quantified using the Phadia CAP System FEIA (Phadia, Uppsala, Sweden).

\section{Patient selection for analysis}

There were 7 children who could not start OIT because of mild to severe allergic reactions to the initial dose on the first day of OIT trial. Four children did not appear either on the first day of the trial or during the subsequent planned visits. We excluded the 11 above-mentioned children from the study population, and statistically analyzed the remaining 71 .

\section{Statistical analysis}

The clinical records of the children were overviewed and classified into two groups according to the outcome of OIT: one group consisted of responders who could reach remission and continued to show progress throughout the following period; another group consisted of poor responders who showed persistent allergic reactions and could not reach the remission stage, or who showed regression and lost desensitization during the maintenance phase after reaching the remission stage. In order to identify what caused the differences between the two groups, we analyzed the outcome of the therapy to see if any of the following factors were significantly different between the above two groups; sex, age at starting OIT, egg-specific IgE level before starting OIT, the initial dose used for OIT, symptoms during the clinical course (digestive, skin, respiratory, otorhinologic), and dislike of eggs. For statistical analysis of the receiver operating characteristics (ROC) of age at starting OIT plots between the two groups, the optimal cut-off values showed 4.5 years of age and therefore we divided the age criteria into two groups; younger and older; that is, over 5 and under 4 . The egg-specific IgE values of class $6(>100 \mathrm{UA} / \mathrm{ml})$ were assigned a value of $101 \mathrm{UA} / \mathrm{ml}$ and class $0(<0.35 \mathrm{UA} / \mathrm{ml})$ was assigned a value of $0.15 \mathrm{UA} /$ $\mathrm{ml}$. The initial dose of OIT was indicated at $1 / 2,1 / 4,1 / 8,1 / 16$ or even smaller partitions (power of 2) of an egg, where a middle-sized raw egg weight were considered to be $64 \mathrm{~g}$. The data with a skewed distribution were analyzed after a logarithmic transformation. We used the SPSS (version 11.0 for Windows) software package, with a multivariate stepwise logistic regression analysis with a forward selection method. The factors included were gender ( 1 for male, 0 for female), age ( 1 for $\geq$ 5, 0 for $\leq 4), \log 2$ (egg-specific serum $\operatorname{IgE}$ ), and $\log 2$ (initial dose), digestive symptoms ( 1 or 0$)$, skin symptoms ( 1 or 0$)$, otorhinologic symptoms ( 1 or 0$)$, respiratory symptoms ( 1 or 0$)$ and dislike of eggs ( 1 or 0 ). $\mathrm{p}$ values of $<0.05$ (by two-tailed tests) were considered to be significant.

\section{Results}

The median age of the 71 children at the initial challenge of OIT was 5 years (IQR, 3 years). There were 27 children under 4 years of age $(38 \%)$ and 44 over 5 years of age (62\%). There were 48 male $(68 \%)$ and 23 female (32\%) children. When the children become tolerant and could ingest half of an egg, we define this phase as remission. The phase before reaching remission is described as tolerance for the processed material. A total of 40 (56\%) children reached remission in the OIT trial and continued to be encouraged to ingest further increased doses. The remaining 31 (44\%) could not reach the remission stage, but were also encouraged to continue ingesting the highest tolerated doses, and were challenged to increase them at regular visits. There was no child who experienced a severe adverse reaction throughout the course. Only antihistamine drugs and corticosteroid ointment were used to treat the symptoms but epinephrine was never used as a rescue-treatment. In all, the children took a median of 213 days (IQR, 225 days) to reach remission. We have followed them up for a median of 340 days (IQR, 323 days) since the initial challenge.

The children were classified into two groups according to their clinical patterns, as shown in Figure 1. One consisted of OIT responders who reached the remission and have continued to ingest further increased doses, while the others consisted of poor responders who could not either reach the remission stage or who showed regression

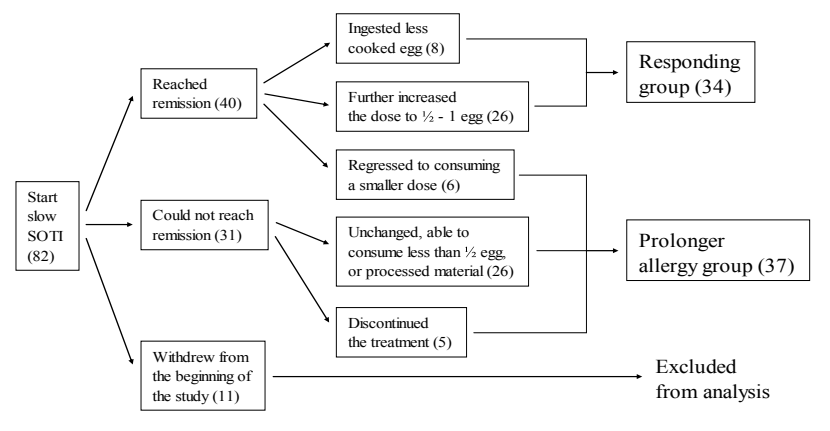

* () shows the numbers of children

Figure 1: Classification according to the clinical patterns of children treated with home-based slow OIT. 


\begin{tabular}{|c|c|c|c|c|}
\hline & & Responding & Prolonged & Total \\
\hline \multirow[t]{2}{*}{ Age } & $\leq 4 \mathrm{yrs}$ & $19(70.4 \%)$ & $8(29.6 \%)$ & $27(100 \%)$ \\
\hline & $\geq 5 \mathrm{yrs}$ & $15(34.1 \%)$ & $29(65.9 \%)$ & $44(100 \%)$ \\
\hline \multirow[t]{2}{*}{ Sex } & female & $12(52.2 \%)$ & $11(47.8 \%)$ & $23(100 \%)$ \\
\hline & male & $22(45.8 \%)$ & $26(54.2 \%)$ & $48(100 \%)$ \\
\hline \multirow[t]{2}{*}{ Digestive symptoms } & no & $23(54.8 \%)$ & $19(45.2 \%)$ & $42(100 \%)$ \\
\hline & yes & $11(37.9 \%)$ & 18 (62.1\%) & $29(100 \%)$ \\
\hline \multirow[t]{2}{*}{ Skin symptoms } & no & $11(39.3 \%)$ & $17(60.7 \%)$ & $28(100 \%)$ \\
\hline & yes & $23(53.5 \%)$ & $20(46.5 \%)$ & $43(100 \%)$ \\
\hline \multirow[t]{2}{*}{ Otorhinologic symptoms } & no & $27(43.5 \%)$ & 35 (56.5\%) & $62(100 \%)$ \\
\hline & yes & $7(77.8 \%)$ & $2(22.2 \%)$ & $9(100 \%)$ \\
\hline \multirow[t]{2}{*}{ Respiratory symptoms } & no & $24(50.0 \%)$ & $24(50.0 \%)$ & $48(100 \%)$ \\
\hline & yes & $10(43.5 \%)$ & $13(56.5 \%)$ & $23(100 \%)$ \\
\hline \multirow[t]{2}{*}{ Dislike of eggs } & no & $30(48.4 \%)$ & $32(51.6 \%)$ & $62(100 \%)$ \\
\hline & yes & $4(44.4 \%)$ & $5(55.6 \%)$ & $9(100 \%)$ \\
\hline Total & & $34(47.9 \%)$ & 37 (52.1\%) & $71(100 \%)$ \\
\hline
\end{tabular}

Table 1: Crosstabulation for the Clinical Course.

\begin{tabular}{|l|l|l|l|l|l|}
\hline & Beta & SE & $\boldsymbol{p}$ value & OR & $\mathbf{9 5 \%}$ Cl for OR \\
\hline Age & 1.448 & 0.582 & 0.013 & 4.255 & $1.360-13.318$ \\
\hline Dose & -0.588 & 0.188 & 0.002 & 0.556 & $0.384-0.804$ \\
\hline Constant & -3.440 & 0.993 & 0.001 & 0.032 & \\
\hline
\end{tabular}

*The factors included were gender, age, egg-specific lgE, initial dose, symptoms during the clinical course

(digestive, skin, respiratory, otorhinologic) and dislike of eggs. Only significan results are shown

*Beta: regression coefficient in the equation

*SE: Standard Error of Beta

${ }^{*}$ OR: Odds Ratio (prolonged/responding)

${ }^{*} \mathrm{Cl}$ : Confidencelinterval

Table 2: Variables Included in the Regression Equation.

after reaching remission, thus resulting in a prolonged sensitivity to eggs. A total of $34(48 \%)$ children belonged to the responding group, and $37(52 \%)$ to the prolonged sensitivity group. The median age was 4 years (IQR, 3 years) in the responding group, and 5 years (IQR, 2 years) in the prolonged group. Table 1 (1 to 7) shows the number of cases of each factor (age, sex, digestive, skin, otorhinologic, respiratory symptoms and dislike of eggs) analyzed in the two groups. With regard to the specific IgE level, the median egg-specific IgE was $7.6 \mathrm{UA} / \mathrm{ml}$ (IQR, 13.6 UA/ml) in the responding group and $15.0 \mathrm{UA} / \mathrm{ml}(\mathrm{IQR}, 22.5$ $\mathrm{UA} / \mathrm{ml}$ ) in the prolonged group. The median initial dose on OIT was $4.0 \mathrm{~g}$ (IQR, 6.0g) in the responding group and $1.0 \mathrm{~g}$ (IQR, $3.0 \mathrm{~g}$ ) in the prolonged group, as shown in Figure 2.

We applied the above-mentioned variables to a multivariate stepwise logistic regression analysis and found that the clinical course of the patients was significantly associated with their age and initial dose of OIT (Table 2). The regression equation obtained by Table 2 was determined as below, where $p$ stands for the probability of belonging to the responding group:

$$
\operatorname{logit}(P)=\log _{e} P= \begin{cases}-3.440-0.588 \log _{2}(\text { intial dose }) & \text { if age } \leq 4 \\ -3.440+1.448-0.588 \log _{2}(\text { intial dose }) & \text { if age } \geq 5\end{cases}
$$

We described the probability curve based on the regression equation above as shown in Fig.3, showing the relationship between an initial dose and the probability of a child belonging to the responding group. Clear differences were found in the two groups. Briefly, if a child over 5 years could ingest about $1 \mathrm{~g}$ as an initial dose, the probability of his/her belonging to the responding group was about $22 \%$, while the same probability was $55 \%$ if he/she was under 4 years of age (Figure 3 ).

\section{Discussion}

In the present study, home-based slow OIT was shown to be more successful for the children with egg allergy less than 4 years of age if they were able to ingest over $1 \mathrm{~g}$ in weight of raw egg as an initial dose. However, we can see that children over 5 years of age must ingest much more egg as an initial dose to obtain successful results using the same method. In order to reach a $50 \%$ success level, we were able to determine how much egg the children are required to ingest as an initial dose by substituting the value $\mathrm{p} / 1-\mathrm{p}=1$ in the above equation, which gives the initial dose required to reach the $50 \%$ borderline as follows:

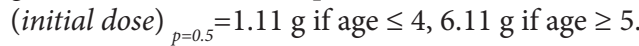

This means that a child over 5 years of age needs to ingest more than $6 \mathrm{~g}$ as an initial dose to reach $50 \%$ success. This dose could be one of the indication criteria to judge whether a child over 5 years of age should be put on home-based OIT or whether other immunotherapies, such as rush OIT, should be used $[1,4,6,8,10-12,14,15]$. However, even if the child is over 5 years of age, as long as severe sensitivity is not present, the child may still have a good chance of overcoming the allergy by home-based OIT. Needless to say, a child under 4 years has a larger possibility of success, even if he/she could only ingest a small amount of egg as an initial dose, as he is only required to ingest about $1 \mathrm{~g}$ as an initial dose to reach the $50 \%$ borderline for success.

Younger children are generally believed to outgrow food allergies by age 3 , although the outgrow rate varies according to different studies $[2,16-20]$. Several studies have pointed out that the outgrow rate was lower than previously reported, and that there were certain populations of children who could not outgrow an allergy on their own $[3,17,20]$. A high antigen- specific IgE level was reported to be one of the predictive factors for egg allergy persistence [3,18,21]. Therefore, it was of interest to elucidate whether slow OIT could alter the natural course of children who have difficulty outgrowing an egg allergy. Ramesh et al. described that it is not very clear whether total avoidance of the specific food increases the chances of outgrowing the allergy, or if the introduction of small amounts increases tolerance [2]. Staden et al. performed a control study to assess the effects of OIT. They commented that OIT substantially increases the threshold dose necessary to elicit allergic reactions, and children who undergo OIT could reach their maintenance dose much faster than the children on an elimination diet. In this respect, OIT is considered to be superior to an elimination diet [12]. Indeed, some control studies have been reported on OIT (rush manner) for older children with a milk allergy, and they found no improvement in an elimination group $[8,22]$. Thus, several studies have indicated that OIT may alter the natural course or increase the threshold dose in children who have difficulty outgrowing their food allergy.

Our study unfortunately lacked a control group, and it is therefore not possible to demonstrate the superiority of home-based OIT to an elimination diet. However, we suggest that leaving children with persistent egg allergy up to 5 years of age without medical intervention may deprive them of chances of overcoming egg allergy by home-based OIT, which seems to be a safer and more practical approach than rush OIT because it does not require hospital admission. Compared with rush OIT, where most of the applicants have experienced adverse reactions $[8,10]$, our home-based slow OIT caused almost no side-effects, except for occasional very mild to moderate symptoms, which did not require adrenaline, intravenous fluids, or oxygen treatment. While many other OIT approaches, including rush OIT, caused reactions at home, the patients under our slow OIT protocol were not bothered by allergic symptoms at home, because the doses of egg were only increased in the outpatient clinic. It is also necessary to point out that the safety of 
$[\mathrm{UA} / \mathrm{ml}]$

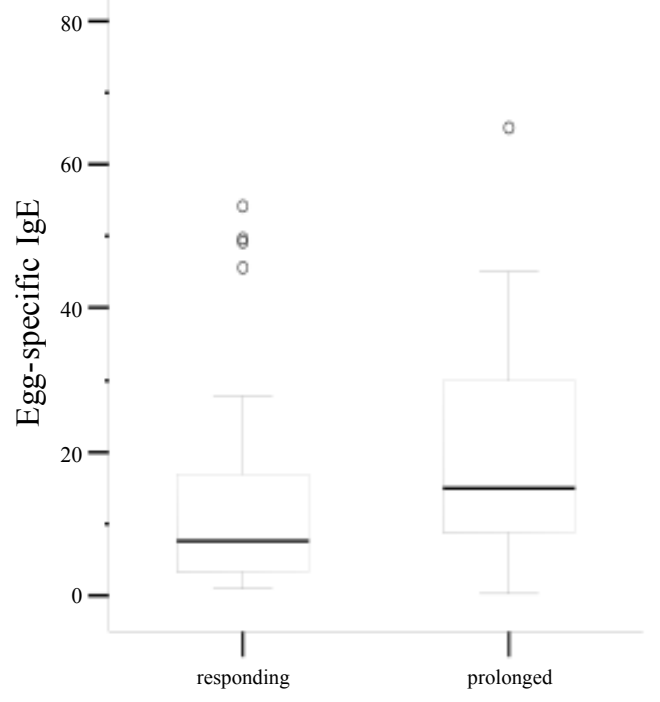

Clinical course $[\mathrm{g}]$

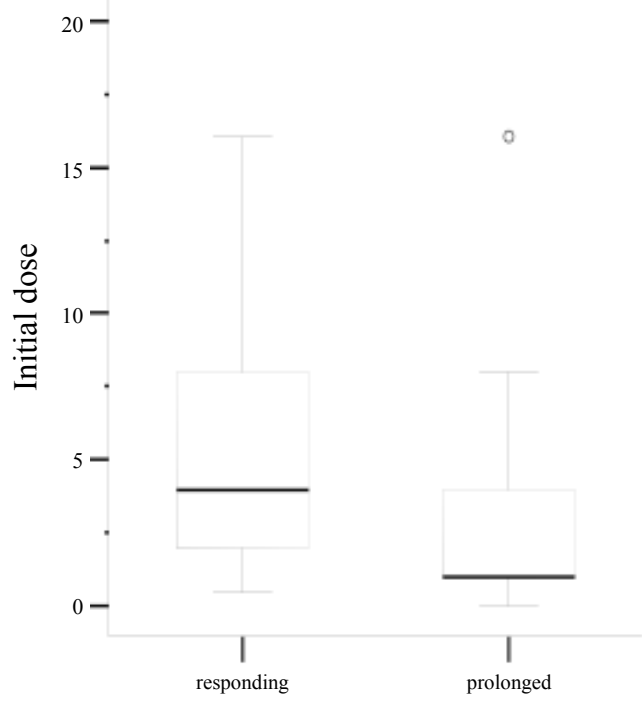

Clinical course

* Horizontal lines, boxes, whiskers, and circles represent median values, interquartile ranges, minimum or maximum values, and outliers, respectively.

Figure 2: Level of specific egg-white lgE (left) and the initial dose used for home-based slow OIT (right) between the responding group and prolonged allergy group.

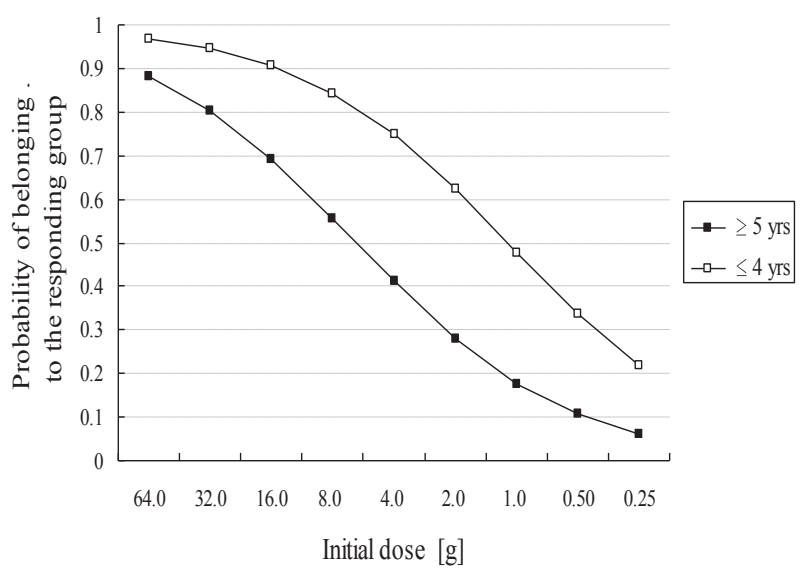

Figure 3: Probability curve for belonging to the responding group.

The relationship between the initial dose and the probability of a child belonging to the responding group between the two groups (over 5 or under 4 years) is shown.

the method makes it possible to treat more patients at the dispensary, while only a few rush OIT patients can be treated because of the need for hospitalization.

In conclusion, home-based slow OIT leads to successful result without any severe allergic reactions when starting OIT before 5 years of age may be critical, because at that time, the children would have a greater possibility of overcoming their egg allergy by home-based OIT, and before they become less likely to be responsive.

\section{Contributions of the Authors}

Conception and design (Kyoko Sudo, Shoichiro Taniuchi), acquisition of data (Kyoko Sudo, Shoichiro Taniuchi, Shindo Okamoto, Yasuko Hatano, Keiji Nakano, Tomohiko Shimo), analysis and interpretation of data (Kyoko Sudo ${ }^{1}$, Shoichiro Taniuchi, Shindo Okamoto, HK), drafting of the manuscript (Kyoko Sudo, Hayato Koshino), critical revision (Kyoko Sudo, Shoichiro Taniuchi, Shindo Okamoto, Hayato Koshino), statistical analysis (Hayato Koshino), and supervision (Shoichiro Taniuchi, Kazunari Kaneko) was done by the respective authors.

\section{Acknowledgment}

This study was supported by the Mami Mizutani Foundation.

\section{References}

1. Kurihara K (2010) Immunotherapy for food allergy. Allergol Int 59: 9-14.

2. Ramesh S (2008) Food allergy overview in children. Clin Rev Allergy Immuno 34: $217-230$.

3. Savage JH, Matsui EC, Skripak JM, Wood RA (2007) The natural history of egg allergy. J Allergy Clin Immunol 120: 1413-1417.

4. Buchanan AD, Green TD, Jones SM, Scurlock AM, Christie L, et al. (2007) Egg oral immunotherapy in nonanaphylactic children with egg allergy. J Allergy Clin Immunol 119: 199-205.

5. Burks AW, Jones SM (2008) Egg oral immunotherapy in non-anaphylactic children with egg allergy: follow-up. J Allergy Clin Immunol 121: 270-271.

6. Burks AW, Laubach S, Jones SM (2008) Oral tolerance, food allergy, and immunotherapy: implications for future treatment. J Allergy Clin Immunol 121 1344-1350.

7. Kulis M, Vickery BP, Burks AW (2011) Pioneering immunotherapy for food 
Citation: Sudo K, Taniuchi S, Takahashi M, Soejima K, Hatano Y, et al. (2013) Key for Successful Home-Based Slow Oral Immunotherapy in Children with Egg Allergy: Age and Sensitivity. J Allergy Ther S3: 009. doi:10.4172/2155-6121.S3-009

Page 5 of 5

allergy: clinical outcomes and modulation of the immune response. Immunol Res 49: 216-226.

8. Longo G, Barbi E, Berti I, Meneghetti R, Pittalis A, et al. (2008) Specific oral tolerance induction in children with very severe cow's milk-induced reactions. J Allergy Clin Immunol 121: 343-347.

9. Clark AT, Islam S, King Y, Deighton J, Anagnostou K, et al. (2009) Successful oral tolerance induction in severe peanut allergy. Allergy 64: 1218-1220.

10. Itoh N, Itagaki Y, Kurihara K (2010) Rush specific oral tolerance induction in school-age children with severe egg allergy: one year follow up. Allergol Int 59: 43-51.

11. Sopo SM, Onesimo R, Giorgio V, Fundarò C (2010) Specific oral tolerance induction (SOTI) in pediatric age: clinical research or just routine practice? Pediatr Allergy Immunol 21: e446-449.

12. Staden U, Rolinck-Werninghaus C, Brewe F, Wahn U, Niggemann B, et al. (2007) Specific oral tolerance induction in food allergy in children: efficacy and clinical patterns of reaction. Allergy 62: 1261-1269.

13. Mukoyama T, Nishima S, Arita M, Ito S, Urisu A, et al. (2007) Guidelines for diagnosis and management of pediatric food allergy in Japan. Allergol Int 56: 349-361.

14. Tang ML (2009) Oral immunotherapy for food allergy. Curr Allergy Asthma Rep 9: 43-49.

15. Barbi E, Berti I, Longo G (2008) Food allergy: from the of loss of tolerance induced by exclusion diets to specific oral tolerance induction. Recent Pat Inflamm Allergy Drug Discov 2: 212-214.

16. Boyano-Martínez T, García-Ara C, Díaz-Pena JM, Martín-Esteban M (2002) Prediction of tolerance on the basis of quantification of egg white-specific IgE antibodies in children with egg allergy. J Allergy Clin Immunol 110: 304-309.

17. de Boissieu D, Dupont C (2006) Natural course of sensitization to hen's egg in children not previously exposed to egg ingestion. Eur Ann Allergy Clin Immunol 38: 113-117.

18. Ikematsu K, Tachimoto H, Sugisaki C, Syukuya A, Ebisawa M (2006) Feature of food allergy developed during infancy (2)--acquisition of tolerance against hen's egg, cow's milk, wheat, and soybean up to 3 years old. Arerugi 55: 533-541.

19. Montesinos E, Martorell A, Félix R, Cerdá JC (2010) Egg white specific IgE levels in serum as clinical reactivity predictors in the course of egg allergy follow-up. Pediatr Allergy Immunol 21: 634-639.

20. Sicherer SH, Sampson HA (2010) Food allergy. J Allergy Clin Immunol 125 S116-S125.

21. Benhamou AH, Caubet JC, Eigenmann PA, Nowak-Wegrzyn A, Marcos CP, et al. (2010) State of the art and new horizons in the diagnosis and management of egg allergy. Allergy 65: 283-289.

22. Skripak JM, Nash SD, Rowley H, Brereton NH, Oh S, et al. (2008)A randomized, double-blind, placebo-controlled study of milk oral immunotherapy for cow's milk allergy. J Allergy Clin Immunol 122: 1154-1160. 